\title{
Neurofibromatosis with Intrahepatic, Retroperitoneal and Pelvic Involvement: A Case Report and Literature Review
}

\author{
Danqing Huang, MS ${ }^{a}$, Min Tang, MS ${ }^{b}$, Aimei Li, MD ${ }^{c}$, Decai Yu, MD ${ }^{d}$, Jun Chen, MD ${ }^{e}$, Min Wu, MS ${ }^{a}$, Wenping \\ Wang, MD ${ }^{f}$, Wentao Kong, $M^{a, *}$ \\ ${ }^{a}$ Department of Ultrasound, ${ }^{b}$ Department of Radiology, ${ }^{c}$ Department of Nuclear Medicine, ${ }^{d}$ Department of Hepatobiliary Surgery, \\ ${ }^{e}$ Department of Pathology, Drum tower Hospital, Medical College of Nanjing University, Nanjing, China; ${ }^{f}$ Department of Ultrasound, \\ Zhongshan Hospital, Fudan University, Shanghai, China.
}

Received November 22, 2020; revision received December 21, 2020; accepted December 31, 2020

\begin{abstract}
Neurofibromatosis type 1 (NF1) is a benign peripheral nerve sheath tumor, which can primarily cause neurocutaneous manifestations. We herein present a rare case of periportal neurofibromatosis with intrahepatic, retroperitoneal and pelvic involvement. The patient underwent abdominal ultrasound (US), magnetic resonance imaging, and a positron emission tomography-computed tomography. Despite these various imaging features, a diagnosis of lymphomas could not be entirely excluded. The patient therefore underwent US-guided biopsy, and a diagnosis of intrahepatic neurofibromatosis was made. Through retrospective analysis of this case along with literature review, we concluded that distributing along the Glisson's sheath may be a characteristic in the diagnosis of NF that involves liver.
\end{abstract}

Key words: Case report; Ultrasonography; Radiology; Neurofibromatosis; Glisson's sheath

Advanced Ultrasound in Diagnosis and Therapy 2022; 01: 029-032

DOI: 10.37015/AUDT.2021.200066

$\mathrm{N}$ eurofibromatosis type 1 (NF1) is a benign peripheral nerve sheath tumor first described by the German pathologist von Recklinghausen in 1882 [1,2]. Then further research elucidated the dominant inheritance of NF1 [3,4]. It is a proliferation of all parts of peripheral nervous system and as a result, it can cause pain, functional damage, and considerable mortality [5-13]. NFs rarely located in abdomen and the involvement of liver is extremely unusual. We herein report a rare case of an 18-year-old male patient with a randomly detected histologically proven neurofibroma of liver with wide infiltrations. This patient underwent abdominal ultrasound (US), magnetic resonance imaging (MRI), and positron emission tomography-computed tomography (PET-CT) with maximum standardized uptake values (SUVmax) accessed successively. The emphasis of this report is on the imaging features and the differential diagnosis of NF occurred in unusual locations, along with related literature reviews.

\section{Case Report}

An 18-year-old male patient was diagnosed as focal liver lesion during the physical examination. The lesion was considered as malignancy according to the imaging features of abdominal ultrasonography and computed tomography (CT). For further evaluation and treatment, he was referred to our hospital.

The patient had no obvious clinical manifestations such as jaundice or hepatosplenomegaly. However, the extremely dark colour of his complexion was noticeable. There was no relevant history of any particular or familial disease. Laboratory findings consisted of normal blood

\footnotetext{
* Corresponding author: Department of Ultrasound, Drum tower Hospital, Medical College of Nanjing University, No 321, Zhongshan road, Nanjing 210003, China

e-mail: breezewen@163.com unrestricted use, distribution and reproduction in any medium provided that the original work is properly attributed.
} 
tests and liver function. Serum carbohydrate antigen 199, carcinoembryonic antigen and alpha-fetoprotein levels showed negative results. The hepatitis B surface antigen was positive while hepatitis $\mathrm{C}$ antibody was negative.

The conventional gray-scale ultrasonography identified heterogeneous hypo-echoic lesion extending along the portal vein. The lesion was measured approximately $14 \times 6.5 \mathrm{~cm}$ (Fig. 1A). The portal vein with mild dilatation was seen within the lesion. The local narrowing of the portal vein compressed by the surrounding lesion was also observed. In colour Doppler flow imaging, the portal vein showed unobstructed (Fig. 1B). After the injection of a dose of $1.2 \mathrm{ml}$ SonoVue as contrast agent, the portal vein and hepatic artery presented well-perfused and locally compressed. The perivascular lesion started to enhance at approximately $20 \mathrm{~s}$ and remained heterogeneous hypo-enhanced in comparison with adjacent liver parenchyma (Fig. 1C, D).

MRI revealed a widely infiltrative mass-like lesion extending along the intrahepatic ducts, with heterogeneous low signal intensity on T1-weighted images and high signal intensity on T2-weighted images.
Notably, areas of low signal intensity surrounded by a halo of high signal intensity could be observed clearly on fat-suppressed T2-weighted images, along with curvilinear structures of low T2 signal (Fig. 2A, B). After the injection of Gd-EOB-DTPA, the portal vein presented serpentine and locally compressed by surrounding lesion but integrally going through the lesion, and the lesion showed hypo-enhancement at this stage (Fig. 2C). During the hepatobiliary phase, the whole lesion presented low signal. Additionally, an unobstructed intrahepatic bile duct could be detected within the lesion (Fig. 2D).

PET-CT showed a huge intra-abdominal, lowattenuation mass with slight-elevated FDG uptake (SUVmax 2.2). The mass encased the intrahepatic vessels (Fig. 3A, B), proliferated along the Glisson's sheath into the liver and extended inferiorly into the retroperitoneum and abdominopelvic cavity. The adjacent vascular system and relevant abdominopelvic organs could not be separated clearly from the lesion. Noticeably, there were multiple cutaneous nodules around the buttock and upper thighs (Fig. 3C, D). The FDG-uptake of both internal and cutaneous nodules were at normal value.
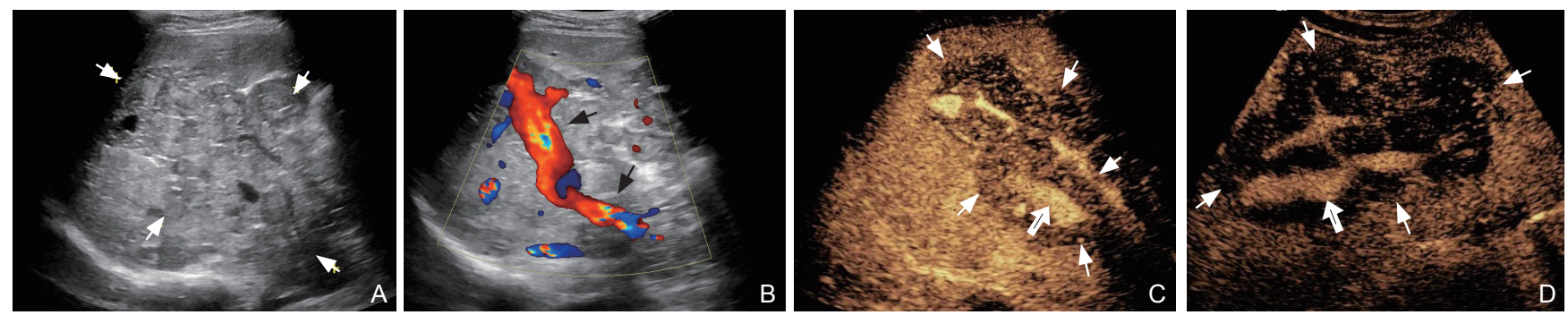

Figure 1 Abdominal ultrasonography. (A) Conventional US showed massive intrahepatic heterogeneous hypo-echoic lesion (arrows) with irregular shape and unclear margin measured approximately $14 \times 6.5 \mathrm{~cm}$; (B) The well-perfused portal vein (arrows) diffusion through the lesion could be observed on CDFI; (C) CEUS demonstrated hypo-enhancement of the perivascular lesion (arrowheads) at portal phase (38s after contrast agent injection), with integrated preservation of the portal vein (open arrow) and hepatic artery (solid arrow); (D) The periportal lesion (arrowheads) remained hypo-enhanced at delayed phase. The portal vein (open arrow) presented well-perfused.
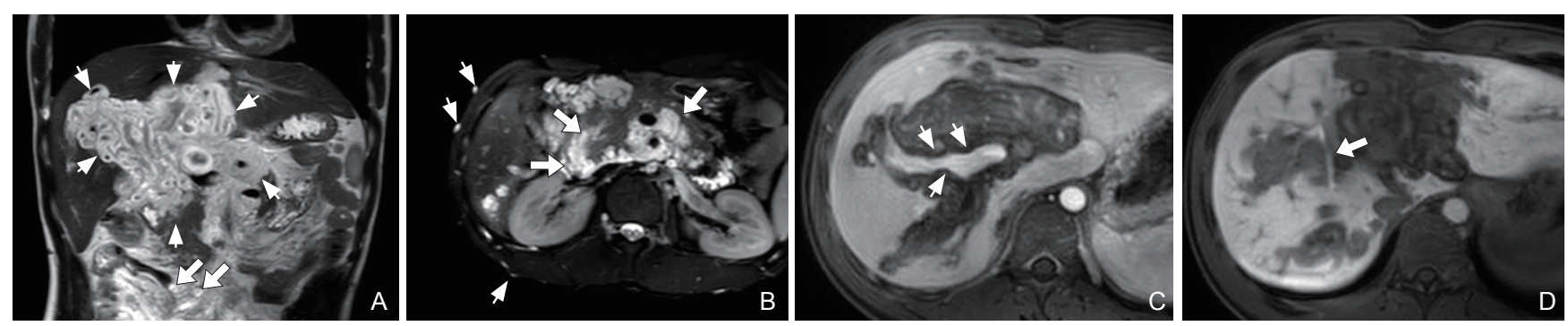

Figure 2 Upper-abdomen magnetic resonance imaging (MRI). (A) Coronal T2-weighted image shows a widely infiltrative high signal intensity masslike lesion (arrowheads) extending along intrahepatic ducts into liver, and proliferating inferior to mesentery (arrows). Central target sign and whorled appearance could be observed; (B) Axial SPAIR shows extensive tumor involvement in the pancreas (arrows). Multiple cutaneous nodules(arrowheads) could also be observed; (C) CE-MRI demonstrates low signal intensity of tumor at portal phase (65s after EOB injection). The portal vein presents serpentine and compressed by surrounding tumor (arrowheads); (D) An unobstructed intrahepatic bile duct (arrow) could be detected at hepatobiliary phase (15min after injection).

US-guided percutaneous liver biopsy was performed with the patient's consent. Hematoxylin and eosin (H\&E) staining showed integrated hepatic vessels surrounded by loosely arranged spindle-shaped cells, which are adjacent to normal hepatocytes (Fig. 4A, B). The histological features favored neurogenic tumors. The immunohistochemical results also presented positive expression for S100 (Fig. 4C) and Sox10 (Fig. 4D), 
along with 1\% markup rate of Ki67 protein. No signs of malignant elements were seen in the biopsy sample. Due to the tumor's wide extension and unclear boundaries with adjacent organs, surgical treatment was not offered. A follow-up after 4 months was operated and there was not much change in MRI images.
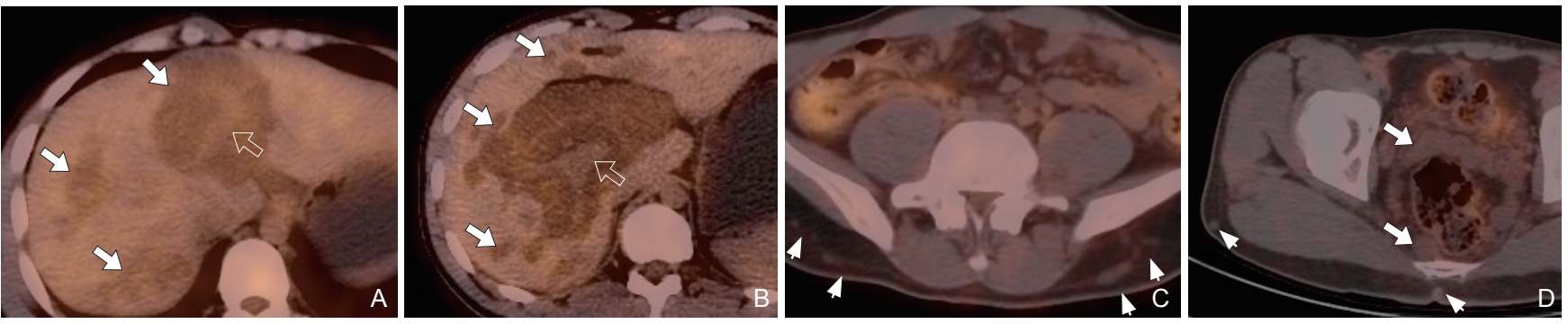

Figure 3 Positron emission tomography-computed tomography (PET-CT). (A, B) PET-CT showed a wide-extended intra-abdominal mass with slight-elevated FDG uptake (solid arrows). The portal vein (open arrow) integrately distributes through the lesion. Multiple cutaneous nodules could be observed around waist and buttock (arrowheads in $\mathrm{C}$ and D). The mass extends inferiorly into the retroperitoneum and pelvic cavity (arrows in D). The FDG-uptake of both internal and cutaneous nodules were minimal.
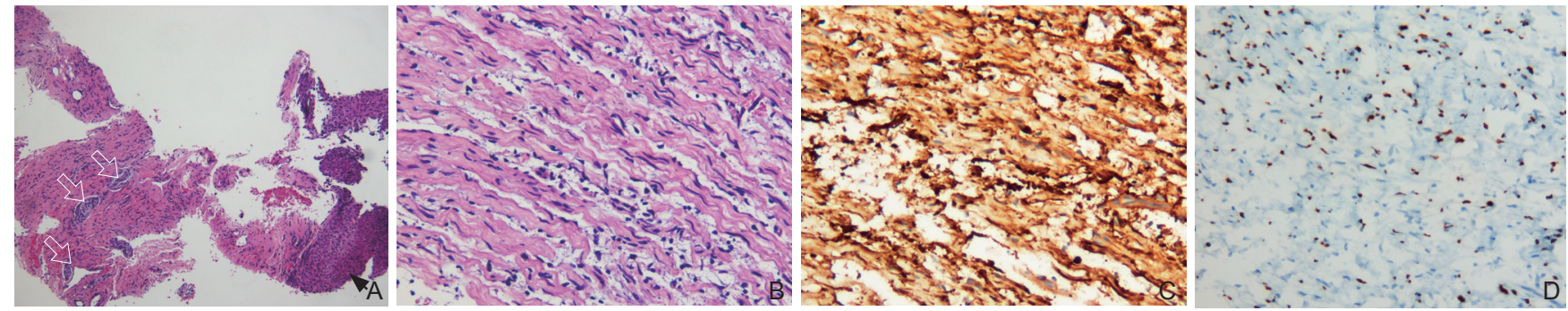

Figure 4 Histological features and immunohistochemical results. (A) Loosely arranged spindle-shaped cells with integrated hepatic vessels distribution (open arrows) and are adjacent to normal hepatocytes (arrowheads) (H\&E stain, 40× magnification); (B) At higher magnification, the sample represents spindle-shaped cells with small, wavy nuclei (H\&E stain, 100× magnification). Positive expression for (C) S100 and (D) So $\times 10$.

\section{Discussion}

NF1 is a benign peripheral nerve sheath tumor, which is autosomal dominant and multisystemic. NF1 primarily cause neurocutaneous manifestations, most of which include cafe-au-lait spots, skin fold freckles, neurofibroma, optic nerve gliomas and lisch nodules on iris $[1,2]$. NFs rarely located in abdomen and the involvement of liver is extremely unusual. It is a proliferation of all parts of peripheral nervous system. However, it has a tendency to transform into malignancy, which is referred to as malignant peripheral nerve sheath tumors (MPNSTs) $[3,4]$. The incidence for NF developing into malignancy is not high but once MPNST formed, it would metastasize widely and cause early death $[3,4]$.

In this case, abdominal US, MRI and PETCT presented conglomerated nodules encasing the intrahepatic tracts, and intrahepatic vessels were found to pass through the lesion integrally. The Glisson's sheath-companying proliferation of the lesion was consistent with the NF's proliferative characteristics, one of which is tending to grow along the length of nerve fibers accompanying vessels and ducts [5-7]. Compared with several previous reports of the hepatic neurogenic tumours, which were biopsy-proven as plexiform neurofibromatosis (PNF) by surgery, those cases shared many common characteristics with our case $[5,6,8$ 13]. According to the report of a mesenteric plexiform neurofibroma analyzed by Matsuki et al., the MRI characteristically presented multiple ring-like structures in T2-weighted images, which performed in our case as central flow-void in liver surrounded by bright structures. We presumed it as the cross-section view of perivascular lesion [9]. Meanwhile, curvilinear hypo-intensity structures on T2 images, which were probably caused by bundles of lemnocytes and collagen fibers, could not be ignored. Those were also described as central target sign and whorled appearances in Delgado et al.'s research of 5 hepatic and pancreatic PNF patients [8]. The radiology performances mentioned above were highly specific and characteristic. However, the pathological samples of our case were obtained by US-guided percutaneous needle biopsy, of which the visible pathohistological structure is limited. There were no signs of fascicles spindle-shaped cells, only loosely arranged cells could be observed. Surgical treatment was not offered because of the wide extension and unclear boundaries with adjacent organs. In addition, the patient showed no obvious symptom and the pathohistological results revealed no malignant elements. The diagnosis of PNF still cannot be excluded.

The contrast-enhanced US and MRI images both showed slight enhancement in either the arterial or the portal phase, which demonstrated hypovascularity of the lesion. In spite of the unvaried 4-month followup MRI images, the NFs still have a chance to develop 
into MPNSTs [1,3,4]. According to previous research, a larger size $(>5 \mathrm{~cm})$ with obscure margins, the presence of calcification, hypervascularity and inhomogeneous areas in the lesion may increase the suspicion of malignancy $[11,15]$. Azizi's research concluded that the value of SUVmax over 3.15 may increase the possibility of malignant transformation. However, considering the great overlap, it is not reliable to assess the malignant tendency by SUVmax [10]. In our case, the SUVmax of the periportal lesion is 2.2 and the SUVmax of internal and cutaneous nodules are within the normal limit.

Although neither destruction of adjacent organs nor embolus in vessels could be observed, as the tumor proliferated along all parts of peripheral nervous system, symptoms caused by the space-occupying compression should be paid real attention to. The patient has been asymptomatic until the second visit. However, it was underlined that NFs involving the hepatic hilum could result in portal hypertension [13]. Thus, close follow-up and related tests are highly recommended. Although the bile duct exhibited unobstructed on the hepatobiliarial phase of CE-MRI at present, a risk of obstructive jaundice should not be neglected. Additionally, since MRI showed obscure boundaries between the mass and gallbladder, pancreas and colonic wall, invasion of the neighboring organs should be paid attention to.

Initially, this patient was considered as lymphomas for the radiological features, while the hypovascularity and low uptake of FDP are inconformity [16]. Therefore, histological biopsy is still indispensable for exclusion. We also noticed that, although this patient had no signs of café-au-lait macules, a generalized hyperpigmentation on his skin and multiple cutaneous nodules around the buttock and upper thighs observed by $\mathrm{CT}$ are also highly suggestive of NF1 [1]. Due to the characteristics of the proliferation and low-attenuation, several neurogenic and periportal tumors should also be considered, such as schwannomas and angiosarcomas $[11,14]$.

\section{Conclusion}

We presented an unusual case of periportal neurofibromatosis with intrahepatic, retroperitoneal and pelvic involvement. The Glisson's sheath-companying proliferation with well-perfused vessels throughout the hypovascular mass may be characteristic which could be detected by imaging techniques. However, considering the rareness of intrahepatic NFs, imaging alone is not sufficient for differential diagnosis, particularly in patients with no signs of NF1. Biopsy is indispensable and the diagnosis should depend on histopathology results. Although the possibility of malignant transformation of NF1 is little, severe space-occupying compression of surrounding organs should be paid real attention to. Considering the active proliferative character, NF1 growing along the Glisson's sheath may lead to portal hypertension or obstructive jaundice. Above-mentioned makes a close follow-up indispensable.

\section{Acknowledgement}

This study was funded by the National Natural Science Foundation of China (81671701)

\section{Conflict of Interest}

The authors declare no competing financial interests.

\section{Reference}

[1] Boyd KP, Korf BR, Theos A. Neurofibromatosis type 1. J Am Acad Dermatol 2009; 61:1-14.

[2] Huson SM, Compston DA, Clark P, Harper PS. A genetic study of von Recklinghausen neurofibromatosis in south east Wales. I. Prevalence, fitness, mutation rate, and effect of parental transmission on severity. J Med Genet 1989; 26:704-711.

[3] Korf BR. Plexiform neurofibromas. Am J Med Genet 1999; 89: 31-73.

[4] Farid M, Demicco EG, Garcia R, Ahn L, Merola PR, Cioffi A, et al. Malignant peripheral nerve sheath tumors. Oncologist 2014; 19: 193-201.

[5] Fujisawa T, Takata M, Ouchi S, Ueyama S, Nakajima T, Mitsutsuji $\mathrm{M}$, et al. Intra-abdominal plexiform neurofibromatosis including periportal, mesentery, and gastrointestinal tract involvement in neurofibromatosis type 1: case report and review of the literature. Clin J Gastroenterol 2011; 4: 292-297.

[6] Rodríguez E, Pombo F, Rodríguez I, Vázquez Iglesias JL, Galed I. Diffuse intrahepatic periportal plexiform neurofibroma. Eur J Radiol 1993; 16: 151-153.

[7] Khandwala K, Sajjad Z, Abbasi SU, Tariq MU. Hepatic, Periportal, Retroperitoneal, and Mesenteric Neurofibromatosis in von Recklinghausen's Disease. Cureus 2018; 10: e2248.

[8] Delgado J, Jaramillo D, Ho-Fung V, Fisher MJ, Anupindi SA. MRI features of plexiform neurofibromas involving the liver and pancreas in children with neurofibromatosis type 1. Clin Radiol 2014; 69: e280-4.

[9] Matsuki K, Kakitsubata Y, Watanabe K, Tsukino H, Nakajima K. Mesenteric plexiform neurofibroma associated with Recklinghausen's disease. Pediatr Radiol 1997; 27: 255-256.

[10] Azizi AA, Slave I, Theisen BE, Rausch I, Weber M, Happak W, et al. Monitoring of plexiform neurofibroma in children and adolescents with neurofibromatosis type 1 by [18 F]FDG-PET imaging. Is it of value in asymptomatic patients? Pediatr Blood Cancer 2018; 65.

[11] Malagari K, Drakopoulos S, Brountzos E, Sissopulos A, Efthimidadou A, Hadjiyiannakis E, et al. Plexiform neurofibroma of the liver: findings on mr imaging, angiography, and CT portography. AJR Am J Roentgenol 2001; 176: 493-5.

[12] Hoshimoto S, Morise Z, Takeura C, Ikeda M, Kagawa T, Tanahashi $\mathrm{Y}$, et al. Plexiform neurofibroma in the hepatic hilum associated with neurofibromatosis type 1: a case report. Rare Tumors 2009; 1: e23.

[13] Lee KH, Yoo SH, Noh GT, Heo WS, Ko BS, Chio JA, et al. A case of portal hypertension by presumed as plexiform neurofibroma at the hepatic hilum. Clin Mol Hepatol 2016; 22: 276-80.

[14] Singh A, Chandrashekhara SH, Handa N, Baliyan V, Kumar P. "Periportal neoplasms"--a CT perspective: review article. Br J Radiol 2016; 89: 20150756.

[15] Lin J, Martel W. Cross-sectional imaging of peripheral nerve sheath tumors: characteristic signs on CT, MR imaging, and sonography. AJR Am J Roentgenol 2001; 176: 75-82.

[16] Barrington SF, Mikhaeel NG, Kostakoglu L, Meignan M, Hutchings M, Müeller SP, et al. Role of imaging in the staging and response assessment of lymphoma: consensus of the International Conference on Malignant Lymphomas Imaging Working Group. J Clin Oncol 2014; 32: 3048-58 